\title{
La plataforma Raspberry Pi como base para la coordinación vertical
}

\author{
Rafael Asenjo, Sonia González, Francisco Corbera, Ángeles Navarro, \\ Andrés Rodríguez, Julio Villalba and Eligius Hendrix \\ Depto. de Arquitectura de Computadores. E.T.S.I. Informática, E.T.S.I. Telecomunicación, \\ Universidad de Málaga \\ \{asenjo,sgn,fjcorbera, magonzalez, andres, jvillalba, eligius\}@uma.es
}

\begin{abstract}
This article describes the process of making use of the interest of students for the Raspberry Pi platform to facilitate study of concepts and techniques in several Engineering courses. Moreover, we show how this platform was used to improve the vertical coordination in the Computer Engineering study from $1^{\text {st }}$ to $4^{\text {th }}$ year in the University of Málaga. The stimulus for this teaching experiment was an educational innovation project PIE13-082 at this university. This project helped to renew the theoretical material and laboratory guides for several courses leading to an improvement of the interest and satisfaction of the students.
\end{abstract}

Keywords: Raspberry Pi, motivation, vertical coordination, renewing laboratory material.

Resumen. En este artículo exponemos cómo hemos aprovechado el interés que los alumnos demuestran por la plataforma Raspberry Pi para facilitar el estudio de conceptos y técnicas impartidas en varias asignaturas de Ingeniería. Además, proponemos usar esta misma plataforma en distintos cursos de forma que se mejore la coordinación vertical en asignaturas de primero a cuarto del Grado en Ingeniería de Computadores de la Universidad de Málaga. El detonante para realizar esta experiencia fue el Proyecto de Innovación Educativa PIE13-082 de la misma universidad. Gracias a este proyecto, se impulsó la renovación de los temarios teóricos y guiones de prácticas de distintas asignaturas lo cual ha resultado en un aumento del interés y en un mayor grado de satisfacción de los alumnos.

Palabras clave: Raspberry Pi, Motivación, Coordinación Vertical, Renovación de Temarios.

\section{Introducción}

Muchos alumnos de ingeniería perciben que las asignaturas de la carrera no son atractivas y están alejadas de su realidad cotidiana. Sin embargo, a la mayoría de estos alumnos les atrae el minicomputador Raspberry Pi (RPi) [16], muy popular en foros tecnológicos relacionados con domótica y robótica. En este trabajo detallamos cómo 
hemos aprovechado el interés que los alumnos ya demuestran por esta plataforma para ponerlo a trabajar en pro de nuestros objetivos docentes: facilitar el estudio de conceptos y técnicas impartidas en varias asignaturas del Departamento de Arquitectura de Computadores de la Universidad de Málaga (UMA). Otro objetivo que se suma al anterior es el de usar la misma plataforma Raspberry Pi para los laboratorios de varias asignaturas de distintos cursos $\left(\right.$ de $1^{\circ}$ a $4^{\circ}$ ), de forma que se mejore la coordinación vertical. Para la consecución de estos objetivos se han elaborado nuevos guiones de prácticas basados en la RPi para varias asignaturas del departamento.

El uso en la docencia de tecnologías familiares al alumno con objeto de incrementar el atractivo de las asignaturas no es algo nuevo. Por ejemplo, en [2] los autores plantean enseñar Sistemas Operativos, pero no usando Windows o Linux como plataforma para las prácticas, sino Android (el Sistema Operativo, SO, usado en casi el 90\% de los Smartphones [3]). Dado que las nuevas generaciones de estudiantes se interesan más por un Smartphone que por un PC, esta estrategia conduce a contenidos más motivadores.

También en [4] se propone potenciar el interés de los alumnos del Grado de Informática en el análisis de los componentes hardware y de su organización para construir un computador moderno mediante el uso de placas basadas en la arquitectura ARM como RPi y Arduino.

La RPi es también la plataforma elegida para motivar la enseñanza de ensamblador en procesadores ARM en [5]. Los autores corroboran en ese trabajo que los alumnos encuentran que el aprendizaje del lenguaje ensamblador es bastante aburrido y tedioso, pero que gracias a la RPi los contenidos se tornan más interesantes y divertidos. Sin embargo, este reciente estudio solo aprovecha la RPi para enseñar una única materia, mientras que nosotros pretendemos que el alumno utilice la RPi como la plataforma de referencia para integrar los distintos conceptos que se van a adquirir en diferentes asignaturas de cursos consecutivos. En esta última línea, en [6] y [7] el objetivo es mejorar la coordinación vertical integrando los distintos conceptos aprendidos en diferentes asignaturas.

En todos estos trabajos mencionados se utilizan distintas tecnologías familiares a los alumnos, pero hasta donde conocemos, ningún trabajo previo considera central el objetivo de la coordinación vertical entre distintas asignaturas al tiempo que se reutiliza la misma plataforma hardware para motivar el aprendizaje de las distintas materias que aparecen en el currículo del Grado de Ingeniería de Computadores.

La coordinación docente vertical es importante para poder desarrollar adecuadamente las competencias de un título y puede contribuir a potenciar el trabajo colaborativo, además de prevenir una formación donde, por ejemplo, se produzcan solapamientos o repeticiones de materia en distintas asignaturas [8], o se fragmente el conocimiento de forma artificial [9]. En [10] se potencia la coordinación vertical para conseguir, entre otros objetivos, que se integren en un solo proyecto, sin solape, los conceptos aprendidos en distintas materias. De forma similar, en [11] se ejercitan las habilidades necesarias para trabajar en grupo mediante proyectos a lo largo de los tres primeros cursos.

Resumiendo, nuestra propuesta destaca sobre los trabajos recientes que acabamos de mencionar en que su finalidad última es triple: i) motivar los contenidos teóricos de 
las asignaturas con prácticas atractivas basadas en RPi; ii) renovar los laboratorios y los guiones de prácticas gracias a una plataforma muy asequible y con una arquitectura de referencia en la era Post-PC; y iii) coordinar verticalmente las asignaturas, de $1^{\circ}$ a $4^{\circ}$, de forma que el alumno construya cada año un proyecto sobre la base aprendida en el curso anterior.

\section{Contexto de la experiencia}

El minicomputador Raspberry Pi [1], o RPi, es una placa del tamaño de una tarjeta de crédito y un precio de alrededor de $30 €$. El objetivo principal de sus creadores, la Fundación Raspberry Pi [12], era promover la enseñanza de conceptos básicos de informática en los colegios e institutos. La plataforma RPi tiene una gran comunidad de usuarios activos, lo que redunda en una gran cantidad de documentación (principalmente en inglés), foros, software libre, aplicaciones, así como ideas de proyectos muy atractivos para los así llamados "makers".

Es patente que, en los alumnos de ingeniería, la plataforma RPi despierta gran interés pues es muy popular en diversos foros tecnológicos. Por ello, en el marco del Proyecto de Innovación Educativa de la UMA PIE13-082, se comenzó una experiencia cuyo objetivo era aprovecharse de ese interés para facilitar el aprendizaje de conceptos y materias impartidas por profesores del Departamento de Arquitectura de Computadores. En el camino, aprovechamos para dar homogeneidad y coordinación vertical entre las prácticas de asignaturas de distintos cursos. Por ejemplo, para el Grado en Ingeniería de Computadores, se hace que un alumno use la RPi para programación en ensamblador en "Tecnología de Computadores" de $1^{\circ}$. A partir de esa base, en $2^{\circ}$ se abordan prácticas más avanzadas de Entrada/Salida en la asignatura "Estructura de Computadores". Al año siguiente, en $3^{\circ}$, se vuelve a usar la misma plataforma para ciertas prácticas de las asignaturas de "Diseño de Sistemas Operativos" y "Arquitectura de Computadores". Por último, en $4^{\circ}$, los alumnos implementarán y programarán un sistema paralelo basado en 4 RPi conectadas en red en la asignatura "Arquitecturas Paralelas". Anteriormente, en cada una de estas asignaturas se usaban herramientas y plataformas muy dispares por lo que cada año el alumno se enfrentaba a un reto nuevo y desconectado de los que superó el año anterior.

Por otro lado, aprovechando que la comunidad de desarrolladores y usuarios de $\mathrm{RPi}$ es principalmente anglo-parlante, en varias de las asignaturas contempladas en esta experiencia se favoreció el uso del idioma inglés. Actualmente existen infinidad de manuales y tutoriales en Internet para usar la RPi como herramienta docente así que es de sentido común aprovechar ese material y redirigir directamente a los alumnos a esas fuentes cuando sea conveniente. Además, en algunas asignaturas (por ejemplo, en uno de los grupos de "Tecnología de Computadores" y de "Estructura de Computadores") las clases de laboratorio se imparten también en ese mismo idioma.

Por último, sabedores de la importancia del trabajo en grupo en las prácticas de asignaturas de Ingeniería, se propuso el uso de herramientas y aplicaciones web que permiten evaluar la participación de cada alumno dentro del grupo. En casi todas las asignaturas contempladas en esta experiencia, los alumnos deben desarrollar aplica- 
ciones software y deben hacerlo de forma colaborativa. Pues bien, herramientas en la web como GitHub o BitBucket simplifican la gestión de versiones y la colaboración durante el desarrollo de aplicaciones. Tanto es así, que muchas empresas que demandan desarrolladores software, en los procesos de selección de personal, solicitan a los candidatos un portafolio con los proyectos alojados en GitHub o BitBucket en los que han participado. Por tanto, usar estas herramientas en la carrera presenta dos ventajas: i) para el alumno, que ganará enteros cuando a la hora de entrar en el mercado laboral se enfrente a un departamento de recursos humanos en un proceso de selección; y ii) para los profesores, ya que estas herramientas, de forma similar a las Wiki's, permiten identificar el grado de colaboración y de esfuerzo que invierte cada alumno al trabajo que se realiza en grupo. Con este último punto, pretendemos que, dentro del grupo de prácticas, la distribución del trabajo esté realmente balanceada, o en caso contrario el profesor lo detectará inmediatamente en la aplicación y tomará medidas para corregirlo.

La Tabla 1 resume las características de las asignaturas implicadas en este trabajo, las cuales pertenecen a las escuelas de Ingeniería Informática, de Ingeniería de Telecomunicación y de Ingeniería Industrial. Para completar este estudio, está previsto incorporar la asignatura de Arquitecturas Paralelas que se imparte en $4^{\text {a }}$ curso del Grado de Ingeniería de Computadores.

Tabla 1. Asignaturas implicadas con implantación evaluada.

\begin{tabular}{llll}
\hline Asignatura & Grado en Ingeniería de & Curso & Num. de alum. \\
\hline Tecnología de Computadores & Computadores, Software, Informática & 1 & 350 \\
Estructura de Computadores & Computadores, Software, Informática & 2 & 200 \\
Diseño de Sistemas Operativos & Computadores & 3 & 25 \\
Arquitecturas Emergentes & Sistemas de Telecomunicación & 4 & 10 \\
Informática Industrial & Tecnologías Industriales & 4 & 10 \\
\hline
\end{tabular}

\section{Descripción de la experiencia}

Aunque en las ramas de ingeniería no es extraño encontrar profesores convencidos de que "el alumno debe venir motivado de casa", nosotros creemos por el contrario que es clave que el profesor haga todo lo posible por hacer atractiva su asignatura. Incluso para alumnos de nivel universitario, impregnar las asignaturas de cierto contenido lúdico es muy beneficioso a la hora de conseguir mejores tasas de éxito académico. Pues bien, la RPi es para un estudiante de ingeniería algo parecido a un juguete, aspecto que los profesores no podemos dejar de aprovechar. También el hecho de que la RPi esté basada en el mismo tipo de procesador ARM que incorporan todos los móviles del mercado, hace que esta plataforma sea atractiva a las nuevas generaciones que han crecido con un Smartphone desde edades muy tempranas.

Sin embargo, los contenidos teóricos y prácticos de gran parte de las asignaturas impartidas en el Departamento de Arquitectura de Computadores de la UMA, han girado en los últimos años en torno a otra arquitectura más tradicional: los procesado- 
res RISC de la familia MIPS. Esto no sólo es así en nuestro departamento, sino también en la mayoría de departamentos de "Computer Science" de Universidades líderes en el mundo. La razón principal es que el libro de referencia [13] usa un MIPS como caso de estudio. Nuestro argumento es que el procesador MIPS no es familiar ni atractivo para los alumnos. Los móviles no llevan MIPS, ni la RPi, ni la mayoría de dispositivos móviles y de consumo que los alumnos ven como tecnología cercana y de uso cotidiano (televisores, electrónica de consumo, etc). Por el contrario, todos estos dispositivos se basan en procesadores ARM y la plataforma RPi es, desde nuestro punto de vista, ideal para enseñar cómo están diseñados estos procesadores y cómo sacarles el máximo provecho. De hecho, los autores de libros de Arquitectura de Computadores, conscientes de esta convergencia hacia los procesadores ARM están cambiando el foco de los textos educativos, reorientándolos hacia estos nuevos procesadores [14], [15].

El Proyecto de Innovación Educativa PIE13-082 concedido por la UMA, sirvió como detonante para renovar gran parte de las asignaturas y laboratorios de nuestro departamento. Primero permitió la compra de $20 \mathrm{RPi}$ a cargo del presupuesto concedido, y segundo sirvió de acicate para sacar a los profesores implicados, de la inercia de asignaturas asentadas y de larga trayectoria. Hay que reconocer que renovar los temarios teóricos y guiones de prácticas de tantas asignaturas no es fácil, pero, como veremos, la satisfacción de los alumnos que ahora usan la RPi como plataforma común para el desarrollo de las prácticas ha merecido la pena.

Los recursos utilizados por más de 15 profesores fueron principalmente la Sala de Coordinación del PIE en el Campus Virtual de la UMA, así como una carpeta compartida en Google Drive. También contamos con la ayuda de dos alumnos que realizaron un Proyecto Fin de Carrera (PFC) y un Trabajo Fin de Grado (TFG). El PFC tuvo como objetivo portar un antiguo manual de prácticas en ensamblador para PC (basado en procesador 80x86 y con Sistema Operativo MS-DOS) a la arquitectura ARM de la RPi. El antiguo manual abordaba contenidos para las asignaturas de "Tecnología de Computadores" y "Estructura de Computadores" del plan a extinguir de Ingeniería Informática. Sin embargo, estas prácticas quedaron en desuso debido a las dificultades técnicas para mantener MS-DOS en los PCs de los laboratorios y a que no son factibles en un SO con permisos de usuario, como Windows XP o Windows 7. Portar las prácticas a la RPi eliminaba los problemas de seguridad y administración de los PCs de laboratorio, al tiempo que actualizaba los contenidos para enseñar la arquitectura ARM. Como parte de este PFC se desarrolló una placa de expansión que se puede conectar a la RPi para visualizar el estado de los pines del GPIO (General Purpose Input/Output) mediante LEDs, provocar interrupciones mediante pulsadores o generar sonido en un zumbador (ver Fig. 1). La memoria de este PFC fue posteriormente completada y extendida ligeramente para convertirse en un manual [16]. Este manual se puso a disposición de la comunidad gracias a la plataforma RIUMA de la UMA, manual que algunos profesores de la Universidad Pablo de Olavide están usando en una asignatura de "Fundamentos de Computadores". 

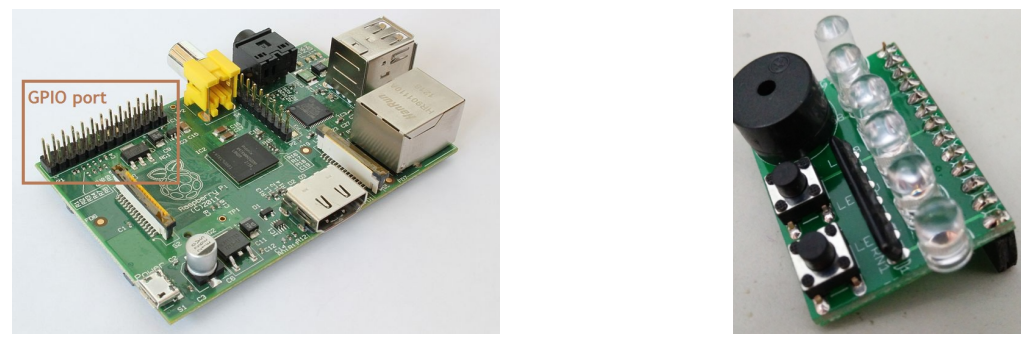

Fig. 1. Placa RPi con puerto GPIO (izq.). Placa de expansión a conectar en GPIO (der.).

La primera mitad del manual está orientada a facilitar el aprendizaje del lenguaje ensamblador para ARM y encaja con las prácticas de la asignatura de "Tecnología de Computadores". La segunda mitad, aborda aspectos de control de la Entrada/Salida (E/S) de la RPi, como son el GPIO y las interrupciones y está más orientada a los contenidos que se imparten en "Estructura de Computadores".

En cuanto al TFG, se desarrolló un planificador de procesos con política Round Robin para $\mathrm{RPi}$, lo que deja abonado el proceso de elaboración de prácticas de laboratorio adicionales para las asignatura de "Diseño de Sistemas Operativos".

Una vez comprobado con este trabajo previo la viabilidad de la RPi como plataforma común de prácticas para un gran número de asignaturas, a lo largo de 2015 y 2016 se compraron más RPi. En la actualidad el departamento cuenta con 172 unidades en total, en parte gracias al reducido precio de este computador.

Con estos precedentes y el material necesario para implantar la RPi como plataforma de prácticas en la mayoría de las asignaturas que imparte el departamento de Arquitectura de Computadores, se han desarrollado un gran número de prácticas y de material docente para las asignaturas recogidas en la Tabla 1, y que describimos en la siguiente sección.

\section{$4 \quad$ Asignaturas implicadas}

\subsection{Tecnología de Computadores, TC $\left(1^{\circ}\right)$}

Tecnología de Computadores es una asignatura obligatoria que se imparte en primer curso de las titulaciones de Grado en Ingeniería Informática, Grado en Ingeniería del Software y Grado en Ingeniería de Computadores. Existen 6 grupos en total de esta asignatura, uno de ellos impartido en inglés. En esta asignatura, partiendo de los conocimientos básicos de electrónica digital adquiridos por el alumno en asignaturas del primer semestre del primer curso, se sientan las bases de la formación en el área de Arquitectura y Tecnología de Computadores, impartiendo los conceptos fundamentales para la comprensión del funcionamiento y diseño de un procesador básico, así como su programación al más bajo nivel. De entre las competencias generales contempladas en esta asignatura, encontramos una única competencia específica que establece como objetivo el "conocimiento de la estructura, organización, funcionamiento e interconexión de los sistemas informáticos y los fundamentos de su programación". De entre los resultados de aprendizaje enfocados a conseguir esta compe- 
tencia, el relacionado con el uso de la plataforma RPi en esta asignatura es: "el alumno deberá ser capaz de realizar programas utilizando el lenguaje ensamblador de una máquina específica".

\section{Metodología.}

Para que el alumno alcance los resultados de aprendizaje especificados, éste debe desarrollar una serie de ejercicios prácticos de programación en ensamblador.

Históricamente la programación en ensamblador se realizaba utilizando el repertorio de instrucciones de un procesador tipo MIPS, que es el procesador utilizado en el libro de referencia por excelencia de estos cursos [13]. Para ello se utilizaba un software que simulaba dicho procesador sobre un PC con Windows. Esto provocaba que el alumno viera de una manera mucho más abstracta y alejada de la realidad este tipo de prácticas.

Con el objetivo de intentar aumentar la motivación del alumno a la hora de realizar las prácticas de programación en ensamblador, en el curso 2014-2015 se impartió un taller de programación en ensamblador sobre la plataforma hardware RPi. Las prácticas se desarrollaron sobre un hardware real y no sobre un simulador, de manera que los alumnos interactuaron directamente con un dispositivo con el que se puede generar sonidos, encender LEDs o accionar pulsadores. Esta interactividad con un sistema real resultó ciertamente incentivadora.

Una vez terminado el taller se sondeó la opinión del alumnado sobre la realización de las prácticas en un dispositivo real (en vez de sobre un simulador). La respuesta del alumnado fue bastante buena, como se puede apreciar en los resultados que mostramos en la sección 5 .

En el curso académico 2015-2016, el equipo docente de la asignatura decidió incorporar de forma obligatoria, un par de prácticas de programación en ensamblador sobre la plataforma $\mathrm{RPi}$, sin abandonar completamente las prácticas de programación basada en un simulador de procesador MIPS. Se les proporcionó nueve vídeos donde se mostraba de forma visual y sonora el resultado que debían conseguir para cada práctica. Además, se le dejaba libertad al alumno para que eligiera al menos dos prácticas de las nueve presentadas. La dificultad de las distintas prácticas no era la misma, por lo que la puntuación asignada a cada una era relativa a dicha dificultad.

Muchos de los alumnos realizaron bastantes más prácticas de las exigidas, e incluso hicieron variaciones sobre las que se les proponían. Entendemos que esto es indicativo del alto grado de motivación que se alcanzó en buena parte del alumnado.

Tras la finalización de las prácticas con las RPi, de nuevo se les pasó a los alumnos una encuesta para que opinaran sobre las mismas, teniendo en cuenta que también habían realizado prácticas en la plataforma antigua. De nuevo el grado de satisfacción de los alumnos con estas prácticas frente a las de la plataforma antigua es muy alto.

Una vez analizadas las ventajas de la nueva plataforma para realizar las prácticas de programación en ensamblador de la asignatura, el equipo docente de la asignatura de Tecnología de Computadores decidió cambiar las prácticas de programación en ensamblador del curso académico 2016-2017 y pasar a realizarlas completamente con la RPi. El tipo de prácticas es muy similar al realizado con la plataforma MIPS, pero cambiando el enfoque al uso de elementos hardware disponibles en la nueva plata- 
forma (LEDs, altavoz y pulsadores), teniendo cuidado para que la complejidad de las prácticas sea básicamente la misma. Aunque entendemos que una de las desventajas del uso de un dispositivo físico frente a un simulador a la hora de realizar las prácticas es la posibilidad de realizarlas fuera del horario de laboratorio asignado a cada grupo, también pensamos que este problema no es muy grave al ser una plataforma bastante asequible para el alumno, además de que nos aseguramos que el tiempo de laboratorio asignado para la realización de las prácticas es suficiente para que el alumno las termine.

\subsection{Estructura de Computadores, EC ( $\left.2^{\circ}\right)$}

Estructura de Computadores es una asignatura obligatoria que se imparte en segundo curso de las titulaciones de Grado en Ingeniería Informática, Grado en Ingeniería del Software y Grado en Ingeniería de Computadores. Existen 5 grupos en total de esta asignatura, uno de ellos impartido en inglés

Esta asignatura parte de los conocimientos básicos impartidos en la asignatura de Tecnología de Computadores, de primer curso, que se ha mencionado anteriormente. En Estructura de Computadores se profundiza en el estudio del subsistema de Entrada/Salida, cuyo diseño y programación se ilustra a través de las prácticas con RPi que se proponen. Tras la realización de las prácticas los alumnos serán capaces de entender el proceso de arranque básico de un procesador, entender los distintos métodos de comunicación de los periféricos con un procesador, así como programar rutinas de tratamiento de interrupción o manejadores en "bare-metal" (sin intervención del SO). Los conocimientos y competencias adquiridos en esta asignatura serán fundamentales para las asignaturas de "Sistemas Operativos" y "Arquitectura de Computadores" que forman parte de la titulación.

\section{Metodología.}

Las prácticas realizadas en EC se organizan en dos tipos de sesiones: 6 sesiones de prácticas guiadas y 6 sesiones de práctica libre. En la primera sesión de prácticas guiadas se les explica el entorno de desarrollo, el sistema de arranque del procesador ARM de la RPi y cómo se compila y carga un programa en la placa para trabajar en modo bare-metal. En las siguientes sesiones, a través de pequeños ejercicios prácticos guiados, se les ilustra cómo funcionan y se programan los distintos métodos de Entrada/Salida en la RPi y cómo interactuar con los LEDs, el altavoz y los pulsadores de la placa de expansión conectada a la RPi en bare-metal.

Tras las sesiones de prácticas guiadas, se propone al estudiante la realización de una práctica final que consiste en la reproducción de una melodía a través del altavoz, acompañada de una función de ecualización (visible a través de los LEDs de la placa de extensión) así como la manipulación de la melodía y ecualizador a través de los pulsadores. Para el desarrollo de esta práctica final los estudiantes disponen de 6 sesiones de práctica libre, durante las cuáles pueden trabajar en parejas. Los estudiantes afrontan esta fase con bastante entusiasmo, porque la práctica es cómo un juego y pueden colaborar entre ellos para ayudarse en el desarrollo y depuración de cada una 
de las funcionalidades de la práctica final. Al finalizar estas sesiones de práctica libre, cada estudiante entrega su práctica final y concierta una entrevista con el profesor/a, que evalúa el nivel de adquisición de los resultados de aprendizaje previstos con el desarrollo de estas prácticas.

\subsection{Diseño de Sistemas Operativos, DSO $\left(3^{\circ}\right)$}

Esta es una asignatura obligatoria de tercer curso del Grado de Ingeniería de Computadores (un solo grupo). Los alumnos de $3^{\circ}$ vuelven a encontrarse con la RPi pero ahora como plataforma para trabajar a bajo nivel dentro del SO Linux (Raspbian). En esta asignatura se pretende profundizar en el diseño de los sistemas operativos, para los que ya se han introducido los conceptos fundamentales en la asignatura de "Estructura de Computadores". Desde un enfoque práctico se estudia cómo se diseñan e implementan los mecanismos y políticas que gestionan, administran y facilitan el uso de los recursos hardware de un computador (procesador, memoria, Entrada/Salida, sistemas de fichero, etc.), función principal del SO.

De entre las competencias específicas que debe adquirir el alumno se encuentran las de "capacidad de diseñar y construir sistemas digitales, incluyendo computadores, sistemas basados en microprocesador y sistemas de comunicaciones" y la "capacidad de diseñar e implementar software de sistema y de comunicaciones". En ambos casos, la plataforma RPi se ha mostrado como una herramienta valiosa para ejercitar y adquirir estas capacidades. Al usarla a lo largo de toda la asignatura, se convierte en clave para obtener los resultados de aprendizaje planteados en la asignatura.

\section{Metodología.}

Se realizaron varias prácticas y proyectos que supusieron la mayor parte de la asignatura. Cada grupo de alumnos fue responsable de su placa de desarrollo (RPi) y pudo, de forma autónoma, recomponerla después de un resultado catastrófico, realizar la depuración o análisis post-mortem necesarios y reconducir su diseño para resolver las contingencias. La facilidad de reinstalación del sistema, simplemente volviendo a escribir el SO en una tarjeta de memoria y restaurando su trabajo en curso desde un servidor de control de versiones Git, permitió que en unos pocos minutos el grupo estuviera de nuevo probando las mejoras y soluciones diseñadas. De esta forma, gracias a la RPi, el alumno no tiene miedo a equivocarse en el diseño, o errar en la implementación, hasta el punto de dejar el sistema inoperativo (irónico, si recordamos el título de la asignatura), ya que la recuperación es indolora. En un PC del laboratorio los alumnos no pueden hacer este tipo de procedimientos delicados que requieren acceso total al sistema (privilegios de administración), puesto que hay muchas posibilidades de dejar el sistema inutilizable con los consiguientes costes asociados a volver a configurar el PC.

Los alumnos más aventajados también tuvieron tiempo de desarrollar un driver para la lectura del sensor de temperatura y humedad DHT11, un reto motivador, puesto que este dispositivo no estaba soportado por el sistema Linux en la RPi. Así que se convirtió en una contribución real de nuestros estudiantes a la comunidad de software 
libre. Este es un desafío algo más importante, porque, aunque el protocolo de comunicación serie con el sensor no es demasiado complejo, debe realizarse de forma eficiente y segura para conseguir lecturas válidas de forma estable.

También destacamos que en esta asignatura es donde por ahora ha tenido mayor sentido usar herramientas de control de versiones.. En particular, se creó una cuenta en BitBucket gestionada por el profesor, que usaron los alumnos durante las prácticas para descargar los materiales, ejemplos y proyectos de partida. A su vez, los alumnos crearon sus propias cuentas en este servidor donde fueron alojando sus proyectos. Cada uno de los proyectos desarrollados tenía como contribuidores a los alumnos que formaban el grupo de trabajo, pero también incluían al profesor, de forma que se podía seguir el desarrollo de los proyectos y comprobar el nivel de implicación y actividad de cada componente del grupo de trabajo.

\subsection{Arquitecturas Emergentes, $\mathrm{AE}\left(4^{\circ}\right)$}

Esta es una asignatura optativa de cuarto curso del Grado de Sistemas de Telecomunicación. En esta asignatura se imparten los conceptos relacionados con arquitecturas avanzadas y emergentes, principalmente relacionadas con las arquitecturas usadas en dispositivos móviles (teléfonos, tablets), sistemas multicore, multiprocesador, procesadores gráficos y arquitecturas heterogéneas. De entre las competencias que debe adquirir el alumno se encuentra la de "entender y explotar el hardware de los computadores y dispositivos móviles".

\section{Metodología.}

La RPi se usó en 2 de las 5 prácticas de la asignatura. Estas dos prácticas se preceden de una explicación de tipo magistral sobre los modos de funcionamiento de la RPi con SO y en bare-metal.

La primera consiste en implementar en ensamblador de ARM algunos códigos en $\mathrm{C}$ de procesado de arrays, así como saber enlazar código $\mathrm{C}$ y código ensamblador, donde este último contenga una versión optimizada a bajo nivel. En dos horas de laboratorio, los alumnos aprenden a conectarse a una RPi con SO Raspbian y a editar, compilar y ejecutar programas ensamblador sobre esa plataforma. Se continúa con un tutorial guiado en el que se enseña cómo hacer que se enciendan/apaguen los leds de la placa de expansión, leer el estado de los botones o generar un sonido, todo ello con soporte de la librería WiringPi. Por último, se les asigna un problema para resolver en el laboratorio (escribir un código que encuentra el valor máximo de un vector) y los ejercicios de procesado de vectores y matrices para resolver de forma no presencial.

La segunda práctica también aborda la programación en ensamblador, pero ahora en modo bare-metal. Esta práctica consume 3 horas de laboratorio y comienza con un tutorial guiado en el que se muestra cómo generar un ejecutable bare-metal mediante compilación cruzada, cuál es el proceso de arranque de la Raspberry Pi y cómo se controlan los leds, el temporizador y el zumbador de la RPi cuando no disponemos del soporte del SO ni de la librería WiringPi. En tiempo de laboratorio los alumnos tienen que resolver un ejercicio en el que deben conseguir que la RPi genere un soni- 
do a $440 \mathrm{~Hz}$ (nota "La" de la escala musical). Como problema a resolver de forma autónoma, no presencial, a los alumnos se les pide implementar un programa ensamblador que reproduce por el altavoz de la placa de expansión el tema "Marcha Imperial" de la BSO de "Star Wars", al tiempo que los LEDs siguen el ritmo de la música.

\subsection{Informática Industrial, II (4 $\left.{ }^{\circ}\right)$}

Esta asignatura es optativa en cuarto curso del Grado de Ingeniería de Organización Industrial. La asignatura aborda la Informática Industrial y las Comunicaciones Industriales desde un punto de vista aplicado.

La competencia específica fundamental de esta asignatura es la de "Adquirir conocimientos aplicados de informática industrial y comunicaciones" y para ello se usa como elemento central la RPi. Esta plataforma nos permite una interconexión fácil con sensores y actuadores que imitarán a un entorno industrial.

\section{Metodología.}

Toda la parte práctica de esta asignatura (50\% de las horas) se ha impartido utilizando la RPi. Se usó la RPi como plataforma de programación en ejercicios de control $\mathrm{y}$ automatización, en los que los alumnos jugaron conectando sensores de temperatura, módulos de radio y de control domótico.

Mediante los GPIOs (entradas/salidas de propósito general) que equipa la RPi y que soportan también diferentes buses de comunicación, se conectaron desde sensores de temperatura hasta módulos de radio de bajo consumo. El uso de la placa RPi permitió la programación e interacción de los alumnos con un sistema completo de control, realizando prácticas de conexión en red y control distribuido.

\section{Evaluación y resultados}

En esta sección presentamos los métodos de evaluación y sus resultados para cada una de las asignaturas presentadas en la sección anterior.

\subsection{Tecnología de Computadores}

La evaluación de la experiencia en TC se realizó a base de encuestas que contestaron los alumnos que realizaron el taller en 2014-2015 y los alumnos que tuvieron que hacer las dos prácticas obligatorias en 2015-2016. De los alumnos que realizaron el taller y contestaron la encuesta, tan solo un $5 \%$ poseían ya una Raspberry, pero el $81 \%$ contestó que se plantearía la compra de una para realizar las prácticas en casa. Esto se ve reflejado en la encuesta del curso 2015-2016 donde el porcentaje de alumnos que poseían una Raspberry ascendió al 28\%, y de nuevo el $82 \%$ considerarían la posibilidad de adquirir una. Consideramos que este aumento puede ser debido, en parte, a que el alumno ya está percibiendo que varias de las asignaturas de su currículum van a utilizar esta plataforma como base para realizar las prácticas (cerca del 90\% de los alumnos consideraban muy interesante que se utilice la misma plataforma en 
distintas asignaturas). Además, se le suma su bajo precio y su versatilidad lo que anima a la compra por parte del alumno. Otro dato a destacar es que a más del $94 \%$ de los alumnos les parecieron muy interesantes estas prácticas, cuestión que ha sido una de las que hemos tenido en cuenta a la hora de decidir cambiar todas las prácticas en el curso 2016-2017 y basarlas en la RPi. Muchos de los comentarios en un campo de texto libre de las encuestas van en el sentido de que a los alumnos les hubiera gustado tener más prácticas con la RPi.

\subsection{Estructura de Computadores}

Se realizó una encuesta de satisfacción de las prácticas basadas en RPi entre los estudiantes de todos los grupos de esta asignatura, EC, al final del primer semestre del curso 2015-2016. Uno de los grupos actuó como grupo de control: en este caso, estos alumnos realizaron las prácticas de programación utilizando una plataforma de desarrollo basada en una FPGA en la que se implementa el MIPS, aunque vieron algunos ejercicios adicionales usando RPi o asistieron a sesiones de laboratorio de otros grupos que utilizaban RPi y pudieron programar en ensamblador de ARM. En total contestaron la encuesta 71 estudiantes, mientras que en el grupo de control recogimos 21 respuestas.

De los 71 estudiantes que realizaron las prácticas en la plataforma RPi y respondieron a la encuesta, el $86 \%$ consideró las prácticas como interesantes o muy interesantes. De hecho, un $75 \%$ cree que con estas prácticas se ilustra bien o muy bien los conceptos de Entrada/Salida que se estudian en esta parte de la asignatura y que son uno de los objetivos fundamentales de estas prácticas. Un $47 \%$ encontró fácil o muy fácil adaptarse al nuevo lenguaje de programación basado en la arquitectura ARM, que es el que se requiere en la RPi. Un $80 \%$ también valora muy positivamente que esta plataforma se utilice en las prácticas de otras asignaturas del Grado. Un 33\% ya tienen una $\mathrm{RPi}$, y de los que no la tienen un $76 \%$ estaría dispuestos a comprársela si se utiliza en otros laboratorios del Grado. En general los alumnos valoraron que se tratara de un sistema real, lo que les permite una inmediata transferencia de conocimiento al mundo profesional. También comentaron que les pareció poco el tiempo dedicado a las prácticas, especialmente el número de horas dedicadas a las sesiones de práctica libre, aspecto que se corrigió en el curso 2016-2017.

En cuanto a los 21 alumnos que participaron en el grupo de control, se obtuvieron porcentajes similares respecto a la percepción del interés de las prácticas y de cómo se ilustran los conceptos que son objetivo de la asignatura. Curiosamente en este grupo, los alumnos parecen más dispuestos a aprender otros lenguajes de programación, no sólo el que necesitan para programar la RPi, sino también otro tipo de plataformas. Recordemos que estos alumnos estuvieron expuestos a varios lenguajes de programación (MIPS y ARM) por lo el esfuerzo fue algo mayor, pero a cambio fueron conscientes del valor añadido que supuso realizar prácticas en varias plataformas y lo valoraron muy positivamente. 


\subsection{Diseño de Sistemas Operativos}

En la encuesta realizada a los nueve alumnos matriculados en DSO el primer año en que se usó la RPi (curso 2014-2015), el 100\% valoró el uso de la plataforma como muy interesante y adecuada. Además, todos ellos opinaron que se debería usar de forma continuada en el resto de asignaturas del área. Muchos de ellos ya tenían o han adquirido una $\mathrm{RPi}$, al final del curso, todos menos uno, poseían una tarjeta propia y la habían usado para poder profundizar en sus desarrollos prácticos fuera del horario de laboratorio. La información sobre la RPi en Internet (principalmente en inglés) permite a los alumnos documentarse y resolver los problemas de diseño y desarrollo planteados.

\subsection{Arquitecturas Emergentes}

La evaluación de la experiencia en $\mathrm{AE}$, se realizó en base a una encuesta a la que contestaron los 5 alumnos matriculados en el curso 2014-2015 (primer año de impartición de la asignatura). Solo uno de los alumnos tenía una RPi en propiedad, pero todos consideraron las prácticas interesantes y formativas. En el campo de texto libre, un alumno comentó: "creo que se le podría dar mucho juego a la Raspberry si ésta se usara desde otras asignaturas, pues se podría ir añadiendo ladrillos en cada asignatura y finalmente conseguir algo más "tecnológico" que hacer sonar una musiquita.". Este comentario nos lleva a reflexionar si los alumnos de $4^{\circ}$ realmente necesitan la motivación/juego con luces y sonidos o ya prefieren actividades más técnicas y profesionales, aunque sean más áridas.

\subsection{Informática Industrial}

Se valoró la introducción de la plataforma RPi mediante una encuesta realizada a los 5 alumnos matriculados en el primer año de impartición de la asignatura (curso 20142015). El 100\% valoró el uso de la plataforma como muy interesante y adecuada. En este caso, tan sólo uno de ellos ya tenía una RPi propia. El perfil de alumno en este Grado, está un poco más alejado del área de las tecnologías de la información y comunicaciones que son los pertenecientes a los Grados de Informática o Telecomunicación. Sin embargo, aceptaron muy positivamente el uso de esta plataforma, que vieron como más atractiva que el clásico PC con el que hasta ahora estaban acostumbrados a trabajar. Los ejercicios de programación y desarrollo en lenguaje $\mathrm{C}$ sobre el entorno Linux (Raspbian) no les resultaron triviales y requirieron de un tiempo de aprendizaje y esfuerzo extra. Sin embargo, el hecho de "tocar" e interactuar con la tecnología, casi jugando, les motivó a seguir intentándolo, mejorando cada vez más sus destrezas y capacidad de manejarse con la programación.

El hecho de trabajar e interactuar con los sensores físicos y realizar el conexionado/cableado de los sensores al interfaz GPIO de la RPi da un valor añadido a la experiencia que la hace muy realista. En los ejercicios prácticos parece que el proceso de creación es más completo cuando además de programar la parte software o inteligencia del sistema, también pueden intervenir físicamente en el diseño y montaje del 
hardware o aparataje necesario. Desde luego, la placa de desarrollo RPi se ha revelado como una opción muy asequible para equipar un laboratorio de programación, control y automatización. Hasta ahora se utilizaban placas de desarrollo equipadas con microcontrolador y diferentes actuadores/sensores en el laboratorio de esta asignatura, pero ahora se combinan con la RPi que ofrece un SO completo y mayor flexibilidad y potencia, de forma que la RPi se convierte en el supervisor de la placa con microcontrolador, dándole además conectividad de red y así poder realizar ejercicios aplicados de IoT (Internet of Things).

\subsection{Resultados Globales}

Aunque se ha hecho una evaluación pormenorizada de cada una de las asignaturas evaluadas en la experiencia, dedicamos este apartado a dar una visión global sobre los resultados de las encuestas de todas ellas. La satisfacción del alumnado que asistió a los laboratorios de estas asignaturas mencionadas se evaluó mediante 9 encuestas anónimas implementadas mediante formularios Google. En total, respondieron 212 alumnos que realizaron las prácticas con RPi. De los resultados de dichas encuestas se desprende claramente que los alumnos disfrutaron con las prácticas propuestas ya que al $89 \%$ de los encuestados las prácticas les parecieron muy interesantes.

La Fig. 2 muestra las estadísticas de dos de las preguntas de dichas encuestas, distinguiendo a los alumnos por curso (de $1^{\circ}$ a $4^{\circ}$ ), e incluyendo la última columna con la media para todos los alumnos de todos los cursos. Las gráficas muestran el porcentaje de alumnos que ha respondido estar "De acuerdo" o "Totalmente de acuerdo" a las preguntas: (a) ¿Te gustaría usar la RPi en otras asignaturas de $1^{\circ}$ a $4^{\circ}$ ?; y (b) ¿Te han parecido motivadoras las prácticas con RPi? El rango de las respuestas contempla 5 opciones ("Total desacuerdo", "En desacuerdo", "Neutral”, "De acuerdo" y "Totalmente de acuerdo").

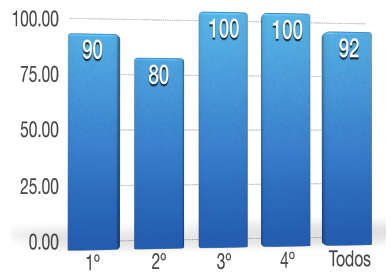

(a)



(b)

Fig. 2. Valoración de los alumnos a las preguntas: (a) ¿Te gustaría usar la RPi en asignaturas de todos los cursos?; y (b) ¿Han sido las prácticas motivadoras?

De estas gráficas se desprende que siempre para más del $80 \%$ de los alumnos (sobre el $90 \%$ de media) las prácticas resultaron motivadoras y se considera que la RPi se debería usar en otras asignaturas de la carrera, dando una continuidad a la plataforma y cierto grado de coordinación vertical.

En la Fig. 3 se muestra el resultado de preguntar por el grado de penetración de la RPi entre los alumnos: qué porcentaje de alumnos ya tienen una RPi en propiedad y, 
de los que no, cuántos estarían "De acuerdo" o "Totalmente de acuerdo" en comprar una.

En todos los cursos más del 15\% de los alumnos ya tiene una RPi, incluso entre los alumnos de primer curso. Destaca el grupo de DSO $\left(3^{\circ}\right)$, donde el $86 \%$ de los alumnos cuentan con una RPi, lo que eleva la media de alumnos que pueden continuar con las prácticas fuera del horario de laboratorio al 39\%. No sorprende que dado el bajo coste de la RPi y la gran cantidad de aplicaciones que se le puede dar (fuera del ámbito académico) que el $77 \%$ de los alumnos se planteara comprar una RPi si se usara en las asignaturas de la carrera.

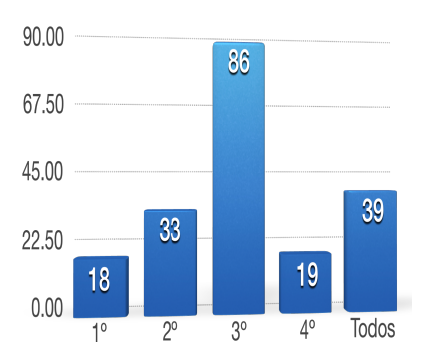

(a)

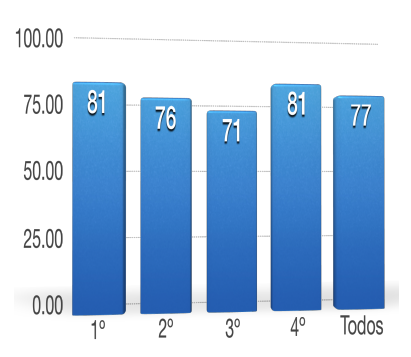

(b)

Fig. 3. Aceptación de la RPi: (a) \% alumnos que ya tienen una RPi en propiedad y (b) \% alumnos que en caso contrario se la comprarían.

Para la asignatura EC se contó con 21 alumnos de control que cursaron las prácticas según el temario de años anteriores usando MIPS en lugar de ARM. De este grupo destacamos que, aunque las prácticas con MIPS eran las obligatorias, el 70\% de los alumnos decidieron voluntariamente hacer también las prácticas con RPi (en algunos casos asistiendo a los laboratorios de otros grupos). El 84\% de este grupo de control también estaba "De acuerdo" o "Totalmente de acuerdo" con que la RPi se usara en el mayor número posible de asignaturas de la carrera.

\section{Conclusiones}

Queremos alumnos motivados en clase y los resultados de este trabajo confirman que la plataforma RPi es un elemento que mejora dicha motivación. Con este objetivo, hemos abordado la renovación de una buena cantidad de prácticas de laboratorio que habían quedado obsoletas y faltas de atractivo para el alumnado. Además, con esta experiencia se ha conseguido actualizar varias de las asignaturas impartidas por el departamento de Arquitectura de Computadores y convencer a los profesores de las bondades de la plataforma RPi a la hora de poner en práctica los contenidos teóricos impartidos en las asignaturas de este departamento. Aunque en las ramas de Ingeniería de Telecomunicación y de Ingeniería Industrial, tenemos menos presencia, esperamos que nuestra experiencia con la RPi sea contagiosa a otros departamentos y áreas. 


\section{Agradecimientos}

El presente trabajo ha sido financiado mediante el proyecto de innovación educativa PIE13-082 de la Universidad de Málaga. Agradecemos a Ma Antonia Trenas ("Estructura de Computadores”), José Ma González (“Arquitectura de Computadores”) y a Javier Hormigo ("Informática Industrial") su trabajo para incorporar, en mayor o menor medida, la RPi como plataforma de prácticas en sus asignaturas. También agradecemos la colaboración de los alumnos que han participado en la experiencia contestando a las encuestas y comentando constructivamente sobre la misma.

\section{References}

1. E. Upton and G. Halfacree, Raspberry Pi user guide, 3rd edition. Wiley, 2014.

2. J. Andrus and J. Nieh, "Teaching Operating Systems Using Android," in Proceedings of the 43rd ACM Technical Symposium on Computer Science Education, 2012, pp. 613-618.

3. "IDC: Smartphone OS Market Share 2016, 2015." [Online]. Available: http://www.idc.com/promo/smartphone-market-share/os.

4. G. Ortega et al., "Procesadores de bajo coste y su aplicación en la docencia de Ingeniería de Computadores," in Actas de las XXII Jenui, 2016, no. 2, pp. 343-349.

5. J. Kawash, A. Kuipers, L. Manzara, and R. Collier, "Undergraduate assembly language instruction sweetened with the raspberry pi," in SIGCSE 2016 - Proceedings of the 47th ACM Technical Symposium on Computing Science Education, 2016.

6. Y.-H. Lu, G. Zhu, and C.-K. Koh, "Using the Tetris game to teach computing," in ASEE Annual Conference and Exposition, Conference Proceedings, 2010.

7. A. Esakia, S. Niu, and D. S. McCrickard, "Augmenting Undergraduate Computer Science Education With Programmable Smartwatches," in Proceedings of the 46th ACM Technical Symposium on Computer Science Education, 2015, pp. 66-71.

8. A. García Martín, Coordinación docente horizontal y vertical. Universidad Politécnica de Cartagena, Servicio de Documentación, 2015.

9. L. Torrego and C. Ruiz, "La coordinación docente en la implantación de los títulos de Grado," Rev. Electrónica Interuniv. Form. Del Profr., vol. 14, no. 4, pp. 31-40, 2011.

10. E. Manchado Pérez and I. López Forniés, "Coordinación por módulos de asignaturas en el Grado de Ingeniería de Diseño Industrial y Desarrollo de Producto de la Universidad de Zaragoza," REDU Rev. Docencia Univ. ISSN 1887-4592, Vol. 10, No. 3, 2012 (Ejemplar Dedic. a Innovaciones en el diseño Curric. los Planes Estud., vol. 10, no. 3, p. 195, 2012.

11. A. Hurst et al., "Towards a Multidisciplinary Teamwork Training Series for Undergraduate Engineering Students: Development and Assessment of Two First-year Workshops," in 2016 ASEE Annual Conference \& Exposition Proceedings, 2016.

12. "Raspberry Pi - Teach, Learn, and Make with Raspberry Pi." [Online]. Available: https://www.raspberrypi.org/.

13. D. A. Patterson and J. L. Hennessy, Computer Organization and Design, 5th ed. Elsevier, 2014.

14. D. A. Patterson and J. L. Hennessy, Computer Organization and Design: The Hardware Software Interface: ARM Edition, 1st ed. Elsevier, 2016.

15. S. L. Harris and D. M. Harris, Digital design and computer architecture.

16. A. J. Villena Godoy, R. Asenjo and F. Corbera, "Prácticas de Ensamblador Basadas en Raspberry Pi.”, Repositorio Institucional de la Universidad de Málaga, RiUMA, 2016. http://hdl.handle.net/10630/10214 